\title{
Adjustment of instantaneous reference walking speed during synchronized robot-based gait rehabilitation
}

DOI 10.1515/cdbme-2017-0011

\begin{abstract}
This paper presents a method to adjust the instantaneous reference speed of the mobile platform or treadmill of robotic gait rehabilitation systems during compliant, synchronized therapy. This method is particularly useful when the robot-patient synchronization algorithms generate compensations that fluctuate significantly throughout the gait cycle, making it unsuitable to apply these compensations directly to the reference speed values. The method was tested with a synchronization algorithm that generates continues temporal-compensations based on a phase controller. The tests comprised simulation of different scenarios and a practical test with a healthy subject using an over-ground gait rehabilitation system.
\end{abstract}

Keywords: gait rehabilitation; human-robot interaction; synchronization.

\section{Introduction}

During the last two decades, several robotic devices have been developed to improve the gait rehabilitation process [1], including treadmill-based systems and mobile devices for over-ground training, among other types. Robotic systems that possess a compliant behaviour (e.g. when using impedance control strategies) allow the patients to deviate from the reference trajectories initially set for the therapy, which may cause synchronization problems between the reference and the actual (patient's) trajectories. To tackle these problems, different synchronization methods have been presented, which include trajectory adaptation algorithms based on the interaction forces between the device and the patient [2], cyclic [3] and continuous [4]

\footnotetext{
*Corresponding author: Santiago Focke Martinez, Institute of Automation, University of Bremen Otto-Hahn-Allee 1, 28359 Bremen, Germany, E-mail: sfocke@iat.uni-bremen.de

Olena Kuzmicheva and Axel Graeser: Institute of Automation, University of Bremen Otto-Hahn-Allee 1, 28359 Bremen, Germany, E-mail: olena@iat.uni-bremen.de (O. Kuzmicheva); ag@iat.uni-bremen.de (A. Graeser)
}

adjustments of the trajectory generator replay speed, and the implementation of adaptive oscillators to adjust the temporal [5] or spatio-temporal parameters [6] of the reference gait patterns, or to adapt the support torque exerted by the system to assist the patient [7].

Many of these synchronization methods are applied in systems that use a treadmill or a mobile platform. However, it is often not specified how the synchronization efforts affect the reference inputs of the controllers that regulate the instant speed of the treadmill or mobile frame. If the online adjustments of the trajectory generation's temporal parameters fluctuate significantly throughout the gait cycle, applying them directly to the inputs of the velocity controllers may cause an undesired oscillatory behaviour.

This paper presents a method to adjust the inputs of the velocity controllers of the mobile platform of the MOPASS system [8] (Figure 1), a robotic device for over-ground gait rehabilitation with active hip and knee joints for motion assistance in the sagittal plane. The presented method was tested with different simulation scenarios and with a healthy subject using MOPASS, running a synchronization algorithm based on the concept presented by Aoyagi et al. in [4]

\section{Synchronization method}

In order to provide the reference trajectories for the system, MOPASS possesses a trajectory generator which creates hip and knee motion profiles depending on its input and on the reference cadence set by the therapist. The input of the generator in an instant $k$ is

$$
t[k]=t[k-1]+\triangle t+\triangle t_{\text {comp }},
$$

where $\Delta \mathrm{t}=1 / f_{c}$ is the time elapsed between two consecutive samples, $f_{c}$ is the sampling frequency of the generator and $\Delta t_{\text {comp }}$ corresponds to a time compensation element (i.e. the synchronization effort).

The value of $\Delta t_{\text {comp }}$ is obtained by means of a phase controller working together with a phase estimator (based on [4]). The latter is in charge of comparing the reference 

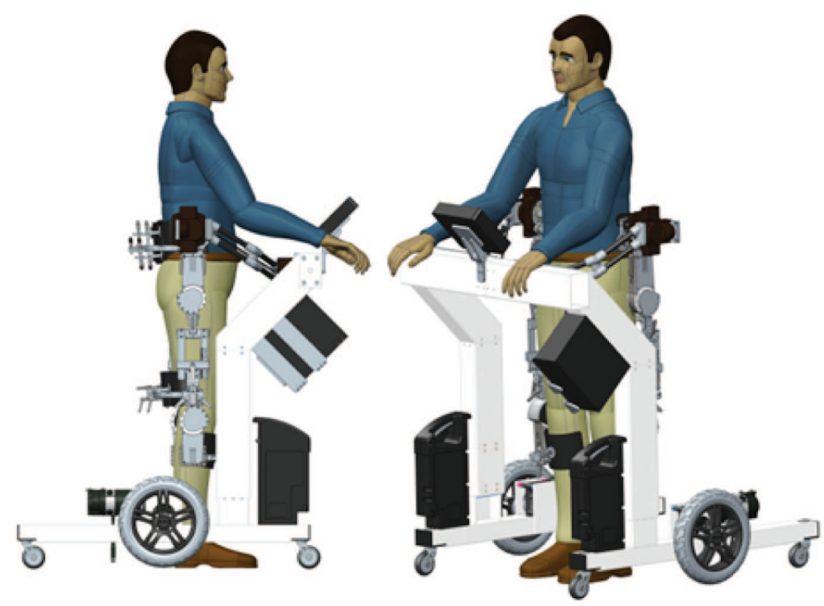

Figure 1: MOPASS system.

gait pattern to the state of the actual gait trajectories, in order to estimate the current phase (i.e. the normalized time or percentage of the stride period) of the patient's gait. The estimated phase $t_{n}{ }^{\star}=[0 \%, 100 \%)$ is obtained by finding the $t_{i}$ that minimizes the cost function

$$
\begin{aligned}
& J\left(t_{i}\right)=\mathbf{W}^{T} \cdot\left[\begin{array}{lllll}
e_{\theta} & e_{h} & e_{d} & e_{t 1}^{2} & e_{t 2}
\end{array}\right]^{T} \\
& e_{\theta}=\sum_{j=1}^{n}\left|\theta_{j, r e f}\left(t_{i}\right)-\theta_{j, a c t}\right| \\
& e_{h}=\left|h_{L, r e f}\left(t_{i}\right)-h_{L, a c t}\right|+\left|h_{R, r e f}\left(t_{i}\right)-h_{R, a c t}\right| \\
& e_{d}=\left|d_{L, r e f}\left(t_{i}\right)-d_{L, a c t}\right|+\left|d_{R, r e f}\left(t_{i}\right)-d_{R, a c t}\right| \\
& e_{t 1}=\left|t_{n}-t_{i}\right| \\
& e_{t 2}=\left|t_{n}^{\star}[k-1]-t_{i}\right|
\end{aligned}
$$

where $N=4$ is the number of joints, $\theta_{j \text {, act }}$ is the actual position of joint $j, \theta_{j, \text { ref }}\left(t_{i}\right)$ is the reference position of joint $j$ at a given normalized time (phase) $t_{i}=[0 \%, 100 \%), h_{p \text {, act }}$ and $d_{p \text {, act }}$ are the actual vertical and horizontal positions of the foot from the leg $p=\{L$ :left, $R$ :right $\}$ with respect to the hip joint calculated from the actual joint angles, $h_{p, r e f}\left(t_{i}\right)$ and $d_{p \text {, ref }}\left(t_{i}\right)$ are the vertical and horizontal positions of the foot of leg $p$ calculated from the reference joint angles at a given normalized time $t_{i}, t_{n}=[0 \%, 100 \%)$ is the normalized time value corresponding to the current input of the generator, $t_{n}{ }^{\star}[k-1]$ is the estimated normalized time (phase) from the previous iteration, and $\boldsymbol{W}$ is the vector of weights. The normalization of the time is done straightforwardly:

$$
t_{n}[k]=\frac{\bmod \left(t[k], T_{r e f}\right)}{T_{r e f}} \cdot 100,
$$

where mod is the modulo operation and $T_{r e f}$ is the reference gait cycle period (i.e. the stride period).

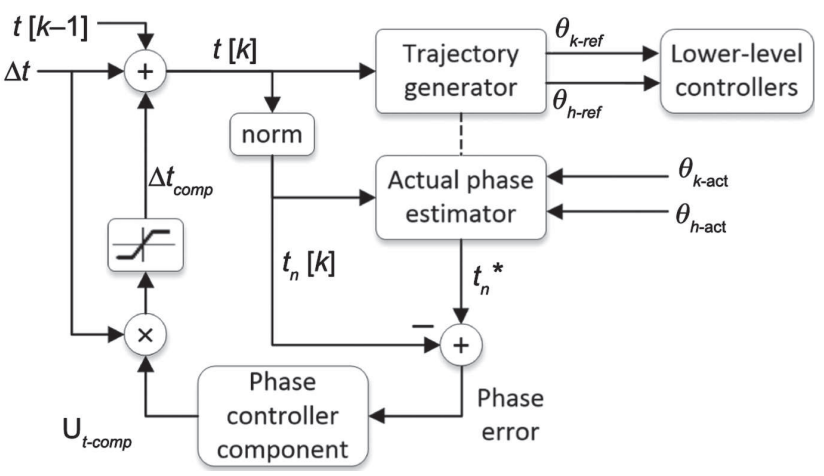

Figure 2: Scheme of the synchronization method.

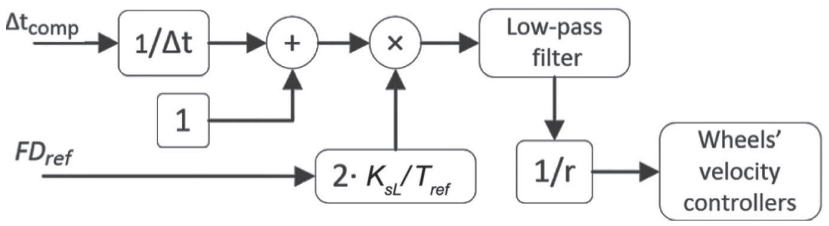

Figure 3: Initial adjustment of the platform speed.

With the estimated value $t_{n}{ }^{\star}$, it is possible to calculate the phase error between the actual and the reference gait patterns. This error is fed to a PI phase controller component in charge of yielding the time compensation

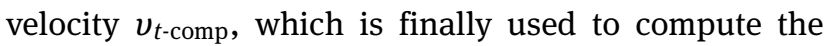
time compensation $\Delta t_{\text {comp. }}$. A simplified diagram of the synchronization process is shown in Figure 2.

\section{Mobile platform speed}

The mobile platform of the MOPASS system is controlled by two drives located in the two motorized wheels. Disregarding the synchronization effort, the inputs to the (angular) velocity controllers in the drives is given by

$$
\omega_{r e f}=\frac{2 \cdot K_{S L} \cdot F D_{r e f}}{T_{r e f}} \cdot \frac{1}{r},
$$

where $F D_{\text {ref }}$ is the total horizontal displacement with respect to the hip joint of the foot along the sagittal axis in a cycle (using the reference trajectories), $K_{S L}=0.85$ is a proportional multiplier and $r$ is the radius of the wheels.

Initially, the time compensation was applied directly to the adjustment of the platform speed using a simple low-pass filter to reduce the oscillations in the speed (Figure 3). However, this solution generated an undesired oscillatory behaviour in the platform speed. To reduce these oscillations, a second approach was taken, (Figure 4), which included some new components. First, a gait phases' detector that identifies the moment of initial contact of the 


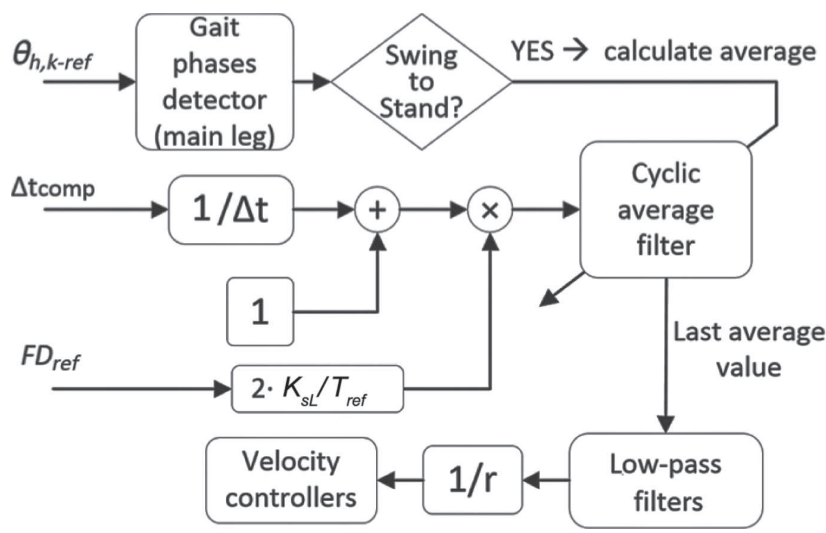

Figure 4: Final adjustment of the platform speed.

main leg (i.e. the one that performed the first step) and, therefore, its swing to stand transition. The second component is a cyclic average filter, which computes the average of the reference platform speed (and updates its output) every time an initial contact is detected. This average value is finally fed to the low-pass filters to smoothen the velocity input of the controllers.

\section{Tests and results}

Initially, the platform-speed adjustment method was assessed in three different simulated scenarios. For the simulations, two different sets of trajectories were used: one was set as the reference gait pattern and the other one as the (simulated) actual one. In scenario $A$, both gait patterns had the same gait cycle period $\left(T_{a c t}=T_{r e f}\right)$; in scenario $B$, $T_{a c t}=0.85 \cdot T_{\text {ref }}$; and in scenario $C, T_{\text {act }}=1.15$ avoid $T_{\text {ref }}$. The simulation results are shown in Figure 5, where the resulting instantaneous platform speeds corresponding to the initial and the improved methods are depicted, together with the corresponding estimated phase error. It can be seen how the inclusion of the cyclic average filter reduced significantly the oscillations in the walking speed, although the system needs more time to react to the phase controller compensations.

A second assessment of the methods was done with a healthy subject performing over-ground walking with the MOPASS system. The subject was asked to walk slowly, then to increase significantly the speed, and finally to reduce the speed and walk slowly again. The test results can be seen in Figure 6, where the platform speed is shown, together with the reference and actual joint trajectories. As with the simulations, a reduction on the oscillatory behaviour of the platform speed can be seen when using the cyclic filter. Moreover, the subject informed that the initial method yielded uncomfortable changes of the speed, whereas the
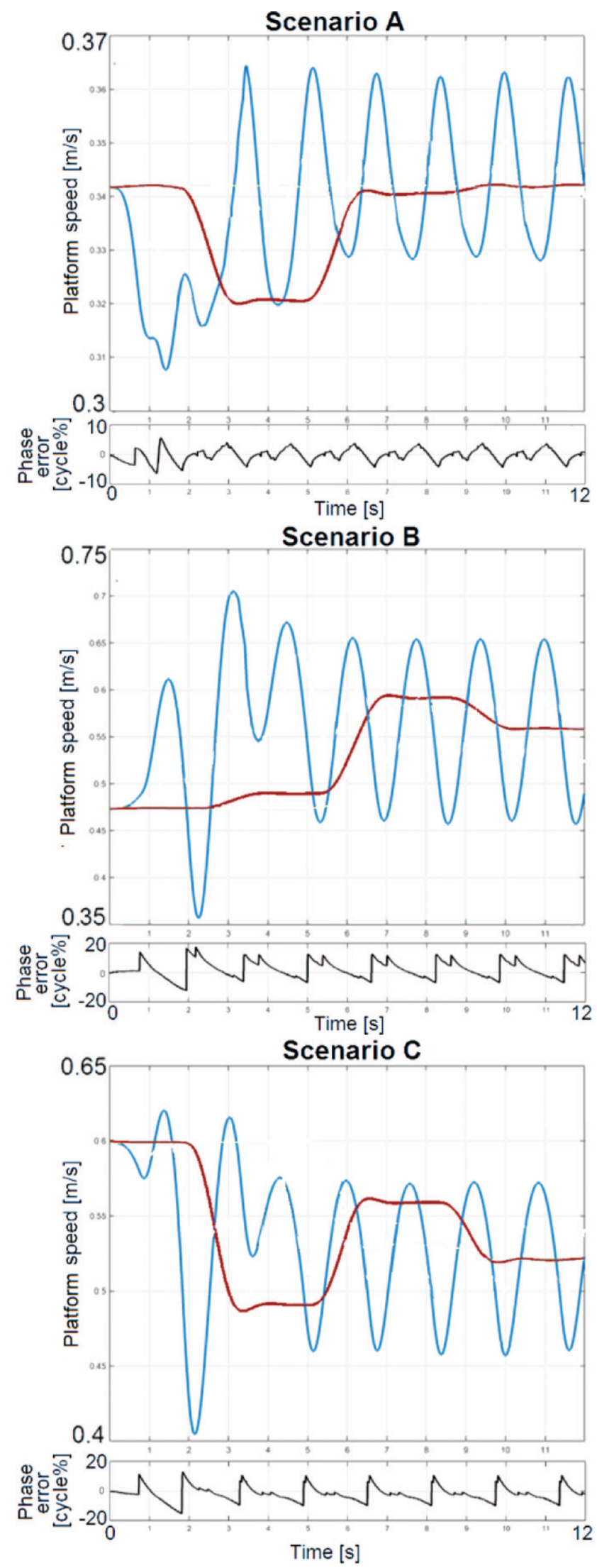

Figure 5: Simulation results. Blue: initial method. Red: improved method. 

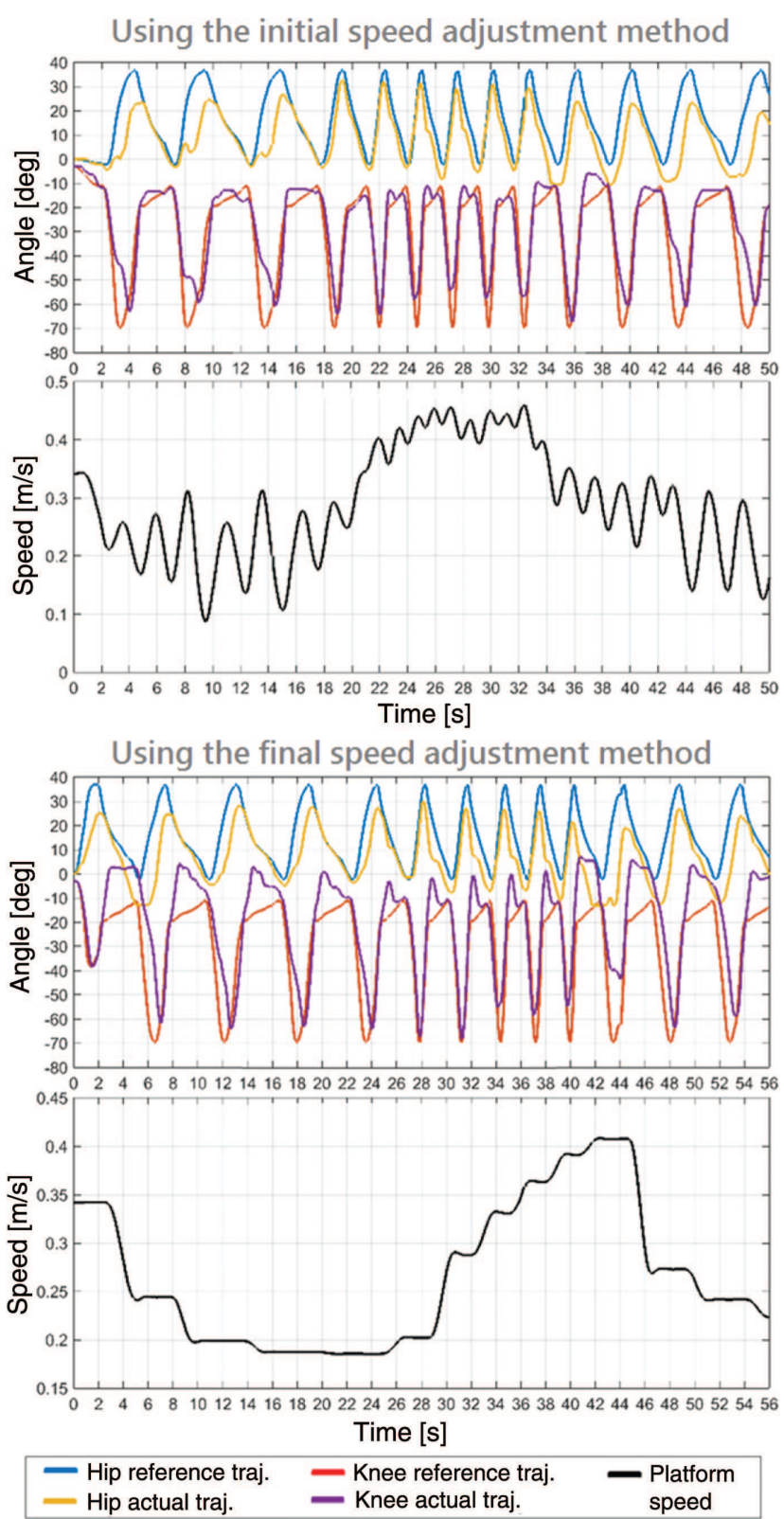

Figure 6: Test results with a healthy subject using MOPASS.

improved method had a much more smooth behaviour during the exercise without feeling a lagged reaction of the platform to the speed changes.

\section{Conclusion}

This paper presented a method to adjust the instantaneous speed used as reference by robotic gait rehabilitation systems (e.g. the speed of the treadmill or mobile frame) during compliant, synchronized therapy. This method is particularly useful when the synchronization algorithms generate compensations that fluctuate significantly throughout the gait cycle. The speed adjustment method was tested with a synchronization method composed by a phase estimator and a phase controller than generates continues temporalcompensations. After carrying out simulations and initial tests with a healthy subject using a mobile rehabilitation device, a reduction of the oscillatory behaviour of the speed was seen, yielding smoother speed profiles that felt more comfortable to the test subject.

\section{Author's Statement}

Research funding: This research was partly supported by the German Federal Ministry of Education and Research as part of the MOPASS (Mobiles, dem Patienten angepasstes, robotergestütztes Gangrehabilitationssystem) project under project number 13EZ1123A-E. Conflict of interest: The authors state no conflict of interest. Informed consent: Informed consent has been obtained from the subject included in the practical tests. Ethical approval: The trials in the MOPASS project received approval from the Ethikkommission der Ärztekammer Bremen.

\section{References}

[1] Koceska N, Koceski S. Review: robot devices for gait rehabilitation. Int J Comp Appl. 2013;62:13.

[2] Jezernik S, Colombo G, Manfred M. Automatic gait-pattern adaptation algorithms for rehabilitation with a 4-DOF robotic orthosis. IEEE Trans Robot Autom. 2004;20:574-82.

[3] Aoyagi D, Ichinose W, Harkema S, Reinkensmeyer D, Bobrow J. An assistive robotic device that can synchronize to the pelvic motion during human gait training. International Conference on Rehabilitation Robotics (ICORR), 2005; Chicago.

[4] Aoyagi D, Ichinose WE, Harkema SJ, Reinkensmeyer DJ, Bobrow JE. A robot and control algorithm that can synchronously assist in naturalistic motion during body-weight-supported gait training following neurologic injury. IEEE Trans Neur Sys Rehabil Eng. 2007;15:387-400.

[5] Chen G, Qi P, Guo Z, Yu H. Gait-event-based synchronization method for gait rehabilitation robots via a bio-inspired adaptive oscillator. IEEE Trans Biomed Eng. 2016.

[6] Ronsse R, De Rossi SMM, Vitiello N, Lenzi T, Koopman B, Van Der Kooij $\mathrm{H}$, et al. Real-time estimate of period derivatives using adaptive oscillators: application to impedance-based walking assistance. In IEEE/RSJ International Conference on Intelligent Robots and Systems, Vilamoura; 2012.

[7] Lenzi T, Carrozza M, Agrawal S. Powered hip exoskeletons can reduce the user's hip and ankle muscle activations during walking. IEEE Trans Neur Sys Rehabil Eng. 2013;21;938-48.

[8] Kuzmicheva O, Focke Martinez S, Krebs U, Spranger M, Moosburner S, Wagner B, et al. Overground robot based gait rehabilitation system mopass - overview and first results from usability testing. International Conference on Robotics and Automation (ICRA), 16-21 May; 2016. 